\section{NP23 (continued)}

Objective: Project goal is to prevent and reduce the prevalence of obesity in Puerto Rican children (1214 years old) through the development of culturally-sensitive-age-appropriate nutrition education materials. General objectives are: generation of knowledge on barriers to healthy eating and physical activity through applied research; development and validation of the nutrition education materials targeting these barriers.

Description: Eight focus groups were conducted, five with 4-H members; two with parents/caregivers and one with Family and Consumer Sciences (FCS) Extension educators, to identify berries in the first objective. Nutrition education materials were developed targeting these barriers. Validation process for the educational materials consisted of three additional focus groups with the $4-\mathrm{H}$ members.

Evaluation: Findings from the focus groups identified four main barriers to healthy eating and physical activity: easy access to unhealthy foods within the school environment; widespread use of sugary foods within the household environment; economic and convenience factors manifested in the preference for fast foods restaurants and sedentary lifestyles due to the high amount of screen time (video games, texting, etc.) Educational materials were developed based on these barriers and included a train-the-trainer manual for FCS Extension educators to be use with their 4- $\mathrm{H}$ clubs. This includes six hands-on-activities and educational videos.

Conclusion and Implications: Findings from the focus groups provided new knowledge and nutrition education resources for Puerto Rican children. The Puerto Rican population is expanding on the US mainland and its cultural idiosyncrasies vary greatly from the Hispanic populations that have been widely studied. Pilottesting the effectiveness of the educational materials and measurements of changes in behavior will be completed during fall 2017.

Funding: 2016-69001-24960

\section{NP24 The Pennsylvania State University Childhood Obesity Prevention Graduate Training Transdisciplinary Program}

Jennifer Savage Williams,PhD, jfs195@psu.edu, Pennsylvania State University, 129 Noll Lab, University Park, PA 16802; Barbara Rolls, PhD, Pennsylvania State University; Kathleen Keller, $P h D$

Objective: This training program prepares pre-doctoral fellows for careers in childhood obesity prevention through transdisciplinary coursework, research, and internships.

Description: This program includes four programmatic areas: nutritional Sciences, child development and family studies, prevention and intervention, and research methodology and statistics.
Evaluation: Evaluation is accomplished through the following: yearly external advisory meetings, program evaluation from trainees, ongoing publications and professional accomplishments of trainees, and biweekly meetings of key personnel.

Conclusions and Implications: Since Fall 2011, we have admitted a total of 19 fellows, nine fellows have graduated; four of those are post-doctoral fellows. Among the nine fellows who have graduated, four are in academia, three are in government and two are in industry positions. Nineteen fellows have produced 93 presentations and 45 peer reviewed publications.

Funding: 2011-67001-30117

\section{NP25 Healthy Caregivers Healthy Children Phase 2 (HC2): Relationship Between Childcare Center Nutrition and Physical Activity Environment and Child Body Mass Index Over One School Year}

Ruby Natale,PhD, PsyD, rnatale@med.miami.edu, University of Miami School of Medicine, 1601, NW 12th Ave, Miami, FL 33136; David Ludwig, PhD, University of Miami School of Medicine; Krystal Sardinas, MPH; Catherina Chang Martinez, MA; Joanne Palenzuela, MA; Sunil Mathew, MA; Sarah Messiah, PhD, MPH

Objective: Despite widespread use of child care centers (CCC) and the high prevalence of obesity among preschool-aged children, most states lack CCC nutrition/ physical activity policies. The Healthy Caregivers, Healthy Children Phase 2 (HC2) project examines the relationship between the CCC nutrition/physical activity environment and child body mass index (BMI) over one school year.

Description: A total of 24 CCCs serving low resource families with $>502$-to-5 year olds attending were randomized to an intervention $(n=12)$ or control $(n=12)$ arm. The HC2 intervention arm CCCs receive implementation of a daily curricula for teachers/parents; children; snack, beverage, physical activity, and screen time policies; and technical assistance with menu modifications. Control arm schools receive an attention control safety curriculum. The Environment and Policy Assessment and Observation tool (EPAO) and child height and weight was collected (Fall/2015, Spring/2016).

Evaluation: Mean child BMI increased in control centers from .43 to .55 while it held relatively consistent (.46 to .49) in HC2 treatment over the school year. EPAO total nutrition score improved in the HC2 treatment centers over the school year (12.4 to 13.5) while no change was seen in control schools (12.5 to 12.6). EPAO physical activity scores remained relatively consistent for both treatment and control centers.

Conclusions and Implications: CCC obesity prevention programs that incorporate curriculum and policies 